\title{
Neue Signale für den Arbeitsmarkt - Zur Akzeptanz gestufter wissenschaftlicher Abschlüsse in der betrieblichen Praxis
}

Hans-Peter Klös

In diesem Jahr muss an deutschen Hochschulen die Umstellung aller Studiengänge auf gestufte Abschlüsse abgeschlossen sein, die Reform der Hochschulausbildung ist damit unumkehrbar. Doch zehn Jahre nach dem Beschluss der europäischen Bildungsminister zur Etablierung eines gemeinsamen Europäischen Hochschulraums ist die sogenannte Bologna-Reform noch einmal zur Chiffre für eine intensive hochschulpolitische Reformdiskussion und für breit angelegte Studierendenproteste geworden. Im Mittelpunkt der Diskussion stehen zum einen die Studienbedingungen an deutschen Hochschulen, zum anderen die immer wieder erneuerte Skepsis gegenüber gestuften Abschlüssen. Neuere Studien zeigen jedoch, dass die neuen Abschlüsse bei Absolventen und Beschäftigern zunehmend auf Akzeptanz stoßen.

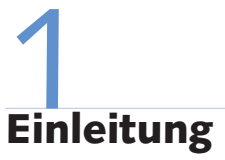

Bei der jüngsten Bologna-Konferenz der Europäischen Bildungsminister im März 2010 wurde Deutschland im „Independent Assessment" mit einem Studierendenanteil in den gestuften Studiengängen von noch unter $50 \%$ zusammen mit sechs anderen Ländern zur Gruppe der Nachzügler gerechnet. Noch nicht abschließend geklärt ist ferner, was mit den Staatsexamen für Juristen und Mediziner geschieht. Zudem ist immer wieder evident, dass es noch ungelöste Probleme gibt: bei den Betreuungsrelationen, bei der Anpassung der Lehrinhalte an die neuen Zeitmuster, bezüglich einer unzureichenden internationalen Mobilität und mit Blick auf fortwährend hohe Studienabbrecherraten (zuletzt Heublein et al. 2010).

Ein Teil der bisherigen Transformationsprobleme ist ganz ohne Zweifel hausgemacht, vor allem weil sich die Durchsetzung einer echten profilbildenden Hochschulautonomie noch als schwierig erweist. Die deutschen Universitäten sind nach wie vor stark staatsanstaltlich geprägt. Bis zu einer autonomen Hochschule, die ihre spezifischen Stärken in Forschung und Lehre zu einem konkurrenzfähigen Profil zusammenführt, um produktiv am Wettbewerb um exzellente Leistungen in Forschung und Lehre teilzunehmen, ist es noch ein vergleichsweise weiter Weg. Damit werden auch wesentliche Erfolgsbedingungen für einen effizienten und effektiven Mitteleinsatz nicht erfüllt (St. Aubyn et al. 2008).
Es gibt aber auch noch andere Gründe für die verzögerte Akzeptanz der neuen Abschlüsse auf beiden Marktseiten: Aufgrund asymmetrischer Informationen haben die Abnehmer der neuen Abschlüsse, also die Einstellenden, bisher nicht durchgängig belastbare Signale über deren Qualität erhalten. Und auch die Absolventen selbst wissen nicht genau, wo sie mit ihren Abschlüssen stehen. Jedoch weisen neuere Befunde auf eine rasch zunehmende Aufgeschlossenheit bei Beschäftigern für die neuen Abschlüsse hin.

Gleichwohl bleiben wichtige strukturelle Herausforderungen für die deutsche Hochschulbildung bisher noch unbeantwortet. Ungeklärt ist, ob und wie sich das Verhältnis der akademischen zur beruflichen Bildung in Deutschland verändern wird. Bekanntlich ist Deutschland eines von wenigen Ländern mit einem sehr hoch entwickelten beruflichen Bildungssystem, durch das noch immer drei von fünf Jugendlichen ihren Weg in eine berufliche Sozialisation nehmen. Nach internationalen Maßstäben erreicht dabei ein Teil der beruflich Qualifizierten in Deutschland Kompetenzniveaus, die andernorts mit akademischen Abschlüssen erreicht werden (Anger/Plünnecke 2009). Unmittelbar damit zusammen hängt die Frage, inwiefern sich durch die gestuften Abschlüsse auch zusätzliche Scharniere zwischen Erstausbildung und berufsbegleitenden Abschlüssen ergeben.

Eine weitere Herausforderung wurzelt in den möglicherweise stark veränderten Kooperationsbeziehungen zwischen Hochschulen und regionalen Akteuren, insbesondere Unternehmen als den
Hauptabnehmern von Hochschulabsolventen. Die strukturellen Veränderungen werden getrieben von dem Oberthema der Nachwuchssicherung, die angesichts demografischer Veränderungen einen zunehmenden Stellenwert erhält und mit Blick auf die Doppeljahrgänge in den Jahren 2012/2013 noch einmal besonders virulent wird. Daraus ergeben sich mit Blick auf den Absolventenbedarf auch neue Formen der Finanzierung von Hochschulen durch Unternehmen, die Ausdruck einer „Corporate Educational Responsibility" sind.

Der Aufsatz gliedert sich entsprechend der vorstehend aufgeworfenen Fragen wie folgt: Nach einer Klärung der theoretischen informationsökonomischen Grundlagen für die Wertigkeit neuer Abschlüsse (Abschnitt 2) werden aktuelle Befunde zu deren Diffusion in die betriebliche Praxis vorgestellt (Abschnitt 3). Anschließend wird diskutiert, wie sich durch die gestuften Abschlüsse das Verhältnis von beruflichen zu akademischen Abschlüssen verändern kann (Abschnitt 4). Abschließend wird der Frage nachgegangen, wie sich Kooperationsbeziehungen zwischen Unternehmen und Hochschule unter dem Einfluss gestufter Abschlüsse ausdifferenzieren können (Abschnitt 5).

Hans-Peter Klös, Dr., Geschäftsführer des Instituts der deutschen Wirtschaft (IW) in Köln und Leiter des Wissenschaftsbereichs Bildungspolitik und Arbeitsmarktpolitik. Arbeitsschwerpunkte: Beschäftigung und Bildung, Demografie und Innovation. e-mail:kloes@iwkoeln.de 


\section{Informationsökonomische Grundlagen}

Für einen Nachfrager von Bildungsinvestitionen besteht ebenso wie für einen Nachfrager von Bildungsabschlüssen grundsätzlich das Problem, die Qualität eines Bildungsangebots nicht stets sachgerecht einschätzen zu können. Weder der Preis noch Informationen über Bildungsmaßnahmen bieten eine hinreichende Grundlage für eine Entscheidung für oder gegen eine konkrete Bildungsmaßnahme bzw. -einrichtung unter Qualitätsgesichtspunkten. Die Einschätzung der Qualität von Bildung wird darüber hinaus von ihrem Investitionscharakter erschwert (Stettes 2006). Ob eine Bildungseinrichtung das versprochene Niveau an Kenntnissen vermittelt hat, zeigt sich erst im Nachhinein, häufig erst im Erfolg oder Misserfolg auf dem Arbeitsmarkt.

Sind die Eigenschaften des Gutes nicht unabhängig von den Eigenschaften des Anwenders, wird eine Dienstleistung oder ein Produkt zu einem „Vertrauensgut“ (Meyer 1990). Um die Informationsmängel bei der Einschätzung des Vertrauensgutes Bildung zu mindern, werden Investoren daher neben den eigenen Suchanstrengungen auch auf die Reputation des Anbieters vertrauen, um eine geeignete Wahl zu treffen (Shapiro 1983). Reputationsmechanismen funktionieren um so eher, je leichter die Qualität der Bildungsmaßnahme eingeschätzt werden kann. Die Bildungsnachfrager werden sich daher vor allem an Informationsquellen orientieren, die für sie eine hohe Glaubwürdigkeit besitzen. Dies sind gegenwärtige oder frühere Teilnehmer der infrage kommenden Bildungsmaßnahmen, Personen aus dem eigenen sozialen Umfeld sowie die Bewertung von Zeugnissen und Zertifikaten durch Dritte.

Abschlüsse und Zertifikate verbessern als funktionierende Signale über die Qualität des Vertrauensguts Bildung die Matching-Effizienz in einer Volkswirtschaft, indem sie Suchprozesse erleichtern. Für den einzelnen Bewerber verringert sich durch Zeugnisse das Risiko, aufgrund persönlicher Merkmale benachteiligt und zum Opfer einer adversen Selektion zu werden. Die Bedeutung der Filterleistung von Zeugnissen, Zertifikaten und Abschlüssen als Qualitätssignale nimmt mit der Nähe zum Arbeitsmarkt zu. Während die berufliche Bildung wegen des dualen Prinzips mit häufiger anschließender Übernahme vielfach den Charakter eines „Inspektionsguts" trägt, weist die tertiäre Bildung an Hochschulen überwiegend Vertrauensgutcharakter auf. Damit kommt den Qualitätssignalen eine herausgehobene Filterleistung zu. Dies wiederum verpflichtet auch die Hochschulen dazu, ihre Filterleistungen glaubhaft darzustellen (Franck/ Opitz 2004).

Gleichzeitig erfüllen Hochschulen auch eine Sortierfunktion (Franck/Opitz 1999), wenn ihre Abschlüsse unterschiedliche Reputation signalisieren. So kann die Differenziertheit der Hochschullandschaft mit ihren mehrstufigen Auswahlprozessen erheblich zur Reduzierung von arbeitgeberseitigen Informationsdefiziten beitragen und ein systematisches Screening durch die Arbeitgeber ermöglichen (grundlegend zum „screening“: Stiglitz 1975). Voraussetzung ist aber unter anderem, dass das „signaling“ als Marktlösung durch Zertifizierungseinrichtungen abgesichert werden kann. Dieses Ziel ist mit europäischen (z.B. ENQA $^{1}$ ) und auch nationalen Akkreditierungsorganisationen (z.B. FIBAA ${ }^{2}$ ) auch im Grundsatz verwirklicht worden.

Trotz dieser Qualitätssicherungsinstitutionen ist die Qualitätsdiskussion bezüglich der neuen Abschlüsse sowohl studierendenseitig wie abnehmerseitig noch nicht durchgängig verstummt. Angesichts asymmetrischer Informationen und des Vertrauensgutcharakters von Hochschulbildung kommt der Validität der mit einem Abschluss verbundenen Qualitätssignale und damit insbesondere der Programm- und Systemakkreditierung durch die Akkreditierungsagenturen sowie deren Supervision durch den nationalen Akkreditierungsrat für die Akzeptanz der neuen Abschlüsse bei Studierenden wie privaten und öffentlichen Arbeitgebern eine herausragende Bedeutung zu.

Zum Teil strittig ist ferner, ob und in welcher Weise Rankings von Universitäten (z.B. Shanghai-Ranking, ${ }^{3}$ CHE-Ranking ${ }^{4}$ ) belastbare Signale für die Qualität der Hochschulen sind. Unbestritten ist die große Beachtung dieser Rankings unter den Hochschulen und Studierenden, die erheblichen Einfluss auf die Studienplatzwahl und die Ausgestaltung des Universitätsbetriebs haben. Gleichzeitig wird die Methodik der Rankings immer wieder kritisiert (Eidgenössisches Staatssekretariat
2009). Vor allem wird angemahnt, bei einer methodischen Weiterentwicklung der bisherigen Rankings auch die Besonderheiten des europäischen Hochschulraums stärker zu berücksichtigen.

Zusammenfassend liefert die Informationsökonomik plausible Erklärungen dafür, dass ein erheblicher Teil der Unsicherheit über die Qualität der neuen Abschlüsse ein informationsökonomisches Phänomen ist: Absolventen ebenso wie deren Beschäftiger können ihre Entscheidungen zur Investition in neue Abschlüsse bisher noch nicht auf langjährige Erfahrungswerte, auf die Qualität der Zeugnisse, auf erworbene Reputation oder auf reputationssimulierende Rankings stützen. Der Übergang zu den gestuften Abschlüssen ist insofern auch als informationsökonomischer Strukturbruch zu bewerten, der erst mit einer quantitativ zunehmenden Verbreitung der neuen Abschlüsse endgültig überwunden werden kann.

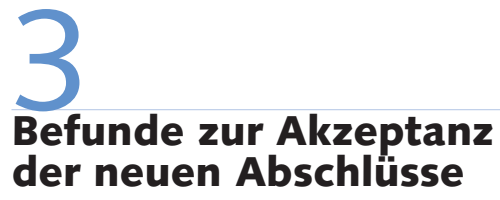

Bei der Rekrutierung zukünftiger Mitarbeiter mit gestuften Abschlüssen stehen private oder öffentliche Arbeitgeber in der Regel also immer noch vor Informationsproblemen. Dessen ungeachtet weisen die Erwartungen der Unternehmen insbesondere an die Bachelorabschlüsse - dies haben auch die verschiedenen „Bachelor Welcome“-Initiativen verdeutlicht - im Kern bereits seit Langem stabile Grundhaltungen auf, wie eine Befragung von 2.135 Unternehmen aus dem Jahr 2007 zeigt (DIHK 2008): Für Unternehmen sind die sozialen und persönlichen Kompetenzen der Absolventen genauso wichtig wie ihr Fachwissen. Teamfähigkeit, Fähigkeit zur selbstständigen Arbeit, Einsatzbereitschaft und Kommunikationsfähigkeit rangieren noch vor dem Fachwissen, das als selbst-

\footnotetext{
1 ENQUA = European Association for Quality As surance in Higher Education.

2 FIBAA = Foundation for International Business Administration Accreditation.

3 Das Shanghai-Ranking ist ein weltweites Hochschulranking, das die Shanghaier Jia-Tong-Universität seit 2003 durchführt.

4 Hochschulranking des Centre for Higher Education Development (CHE)
} 
verständlich vorausgesetzt wird. Zusätzlich sollten Absolventen ihr erlerntes Wissen problemorientiert einsetzen und in den Arbeits- und Kommunikationsprozess im Unternehmen einbringen können.

Darüber hinaus ist Praxisbezug für die Unternehmen von zentraler Bedeutung. Die anwendungsorientierte Vermittlung der theoretischen Kenntnisse und die Integration von Praktika in die Bachelorund Masterstudiengänge werden erwartet. Diesbezüglich sahen Unternehmen in der DIHK-Befragung noch Handlungsbedarf auf Hochschulseite. Sie bemängelten, dass die Absolventen nicht in der Lage wären, ihr theoretisches Wissen in der Berufspraxis umzusetzen. Praxisnahe Studiengänge und die praxisnahe Ausbildung an Berufsakademien und Fachhochschulen (FH) genießen deshalb eine hohe Wertschätzung bei Unternehmen. Absolventen dualer Studienangebote, die ein wissenschaftliches Studium mit der praktischen Arbeit im Unternehmen verzahnen, werden von der Wirtschaft besonders geschätzt.

$\mathrm{Zu}$ Beginn des Wintersemesters 2009/10 sind inzwischen etwa $80 \%$ der Studienangebote auf Bachelor- und Masterstudiengänge umgestellt, darunter in überproportionalem Maße die Fachhochschulen, von denen im Wintersemester 2009/10 bereits rund 96\% der Studiengänge umgestellt waren (HRK 2010). Bei den Absolventen allerdings entfielen im Sommersemester 2009 erst $20 \%$ der Absolventen auf die gestuften Abschlüsse. Gemessen an diesen Eckdaten deuten einige neuere Befunde für die Beschäftiger- wie für die Absolventenseite eine im Grundsatz positive Entwicklung an:

(1) Im Oktober 2009 hat das Internationale Zentrum für Hochschulforschung (INCHER) an der Universität Kassel eine umfassende Studie zum Verbleib von Hochschulabsolventen vorgelegt. Darin wurden die Bildungs- und Berufswege von 36.000 Absolventen deutscher Hochschulen aus 48 Hochschulen eineinhalb Jahre nach ihrem Abschluss untersucht. Dies ist damit die größte jemals durchgeführte Absolventenstudie in Deutschland. Nicht teilgenommen haben die Hochschulen in Bayern, Rheinland-Pfalz und Sachsen, da dort eigene Absolventenbefragungen durchgeführt werden. Etwa $15 \%$ der befragten Absolventen haben einen Bacheloroder Master-Abschluss erworben (3.500 resp. 1.400).
Die Ergebnisse der Studie deuten insgesamt auf eine gute Performanz der neuen Abschlüsse hin (Schomburg/Teichler 2009, S. 115ff.): Bachelor-Absolventen haben gute Berufschancen und bei fächerübergreifender Betrachtung vergleichbare Chancen, eine Anstellung zu finden, wie Absolventen früherer Studiengänge. Die Beschäftigungssuche dauert etwa genauso lang wie bei Diplom- und Magisterabsolventen. Darüber hinaus schätzt sich ein gleich hoher Anteil von Bachelor-Absolventen als ausbildungsadäquat beschäftigt ein und ist mit der Erstanstellung überwiegend zufrieden. Außerdem erweisen sich bei fächerübergreifender Betrachtung insbesondere die Bachelor-Absolventen der Fachhochschulen international mobiler als bislang angenommen.

(2) Deutliche Akzeptanzverbesserungen zeigen sich im speziellen Arbeitsmarkt für Ingenieure, in dem es durch die sogenannte TU 9-Initiative lange Zeit zu einer grundständigen Ablehnung der gestuften Abschlüsse und der damit einhergehenden Ablösung des Titels Diplomingenieur gekommen war. Inzwischen ist die bis zum Jahr 2010 geforderte Umstellung auf Bachelor- und Masterabschlüsse in über neun von zehn ingenieurwissenschaftlichen Studiengängen abgeschlossen. Seit Beginn der Umstellung auf Bachelor- und Masterstudiengänge im Jahr 2000 haben kumuliert betrachtet aber bisher erst 3,5\% der Absolventen in den Ingenieurwissenschaften einen Bachelor- und 5,2\% einen Masterabschluss erworben. Innerhalb dieses Zeitraums kamen etwa zwei Drittel aller Ingenieurabsolventen von der Fachhochschule, ein Drittel kam von technischen Universitäten und Hochschulen. Die Erfahrungsbasis der Unternehmen ist demzufolge noch sehr schmal.

Eine Unternehmensbefragung aus dem Frühjahr 2009 im Rahmen der 10. Welle des IW-Zukunftspanels bei 1.750 Unternehmen aus dem Verarbeitenden Gewerbe und ihren Verbundbranchen, die Ingenieure beschäftigen, deutet auf eine generell hohe Akzeptanz der neuen Abschlussformen in der Wirtschaft hin (KonegenGrenier/Koppel 2009). Mittlerweile sind in jedem siebten Unternehmen Absolventen eines Bachelor- oder Masterstudiengangs der Ingenieurwissenschaften beschäftigt, auch wenn deren Anzahl im Vergleich zu den Diplomingenieuren noch sehr niedrig ausfällt. Die befragten Geschäftsführer und Personalverantwortlichen sehen für Bachelor- und Masteringenieure Einsatzmöglichkeiten in nahezu allen betrieblichen Arbeitsfeldern. Dies gilt ebenso für forschende und innovative Unternehmen. Der Bachelorabschluss führt keineswegs zwangsläufig zu einem Gehaltsnachteil gegenüber Diplomingenieuren von Fachhochschulen und Universitäten. Nachteile im Einstiegsgehalt sind bei der großen Mehrheit der Unternehmen nach drei bis fünf Berufsjahren ausgeglichen.

(3) Schließlich gibt es auch neueste Befunde zu den Erfahrungen der Bachelorstudierenden mit Studium und Lehre unter den neuen Bedingungen. Eine im Februar 2010 vorgelegte Befragung von 17.000 Studierenden zwischen dem Wintersemester 2006/2007 und dem Sommersemester 2007 durch den Studierendensurvey der Arbeitsgruppe Hochschulforschung der Universität Konstanz (Bargel et al. 2010) zeigt ein differenziertes Bild: Es gibt eine große Mehrheit für die Ziele des BolognaProzesses, die Studieneffizienz genießt einen hohen Stellenwert, die Wichtigkeit von Arbeitserfahrungen und Praxisbezug tritt deutlicher denn je hervor und es kann bei Bachelor-Studierenden keineswegs ein generell geringeres Interesse am Forschungsbezug der Lehre und an Forschungserfahrungen im Studienverlauf unterstellt werden als etwa bei DiplomStudierenden. Deutlich werden aber immer noch eine schlechte Gliederung des Stoffes, fehlende Transparenz bei hohen Leistungsanforderungen, eine unzureichende Modularisierung, organisatorische Mängel in der Lehre, ein geringer Kontakt zu den Lehrenden wie auch Defizite bei der Berufsvorbereitung und Berufsbefähigung.

Zusammenfassend kann man eine zunehmende Akzeptanz der neuen Abschlüsse bei den Unternehmen und eine deutliche Aufgeschlossenheit für die Ziele der Reformen auch bei den Studierenden konstatieren. Die aktuellen Bemühungen in der Politik, die Studienbedingungen für die Studierenden durch Investitionen in die Lehre, eine Verbesserung des Stipendiensystems und durch eine Weiterentwicklung der curricularen Inhalte zu verbessern, deuten auf die bisherigen Schwachpunkte der Reform hin, ohne diese aber im Kern infrage zu stellen. Verdichten sich zudem die Befunde über eine zunehmende Ver- 
besserung der Akzeptanz der neuen Abschlüsse bei den Unternehmen, so dürfte mehr als zehn Jahre nach Beginn der Reform auch Deutschland im Europäischen Hochschulraum angekommen sein.

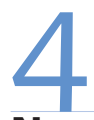

\section{Neue Scharniere zwischen Berufsbildung und Hoch- schulbildung}

Bereits seit Längerem verweisen nationale Berichtssysteme wie etwa der Berufsbildungsbericht und der Nationale Bildungsbericht auf eine schon „traditionelle“ Abschottung der verschiedenen Teilbereiche des Bildungssystems (BMBF 2007). Gerade die Durchlässigkeit der dualen Berufsausbildung in den tertiären Bildungssektor steht daher im Fokus, so etwa bei der ANKOM-Initiative des BMBF zur „Anrechnung beruflicher Kompetenzen auf Hochschulstudiengänge", in der Wege zur Anerkennung von in Aus- und Weiterbildung oder im Beruf erworbenen Kompetenzen bei Hochschulstudiengängen entwickelt und der mögliche Umfang einer Anerkennung im Hinblick auf die Leistungsanforderungen des jeweiligen Studiengangs geprüft werden.

Zu begrüßen ist daher die Rahmenvereinbarung der Kultusministerkonferenz (KMK 2009), in der einheitliche Standards für den Hochschulzugang für Berufspraktiker festgelegt wurden. So sollen Inhaber bestimmter Aufstiegsfortbildungen, wie Meister oder Techniker, eine allgemeine Hochschulzugangsberechtigung erhalten. Ein fachgebundener Hochschulzugang ist für Absolventen vorgesehen, die eine mindestens zweijährige Berufsausbildung verbunden mit einer mindestens dreijährigen fachnahen Berufspraxis vorweisen können. Die Bundesländer sind nun aufgefordert, die weitreichenden Möglichkeiten der Rahmenvereinbarung zügig in Landesrecht umzuwandeln.

Ein durchlässigeres Bildungssystem kann dabei zur Effizienzsteigerung und zur Höherqualifizierung der Bildungsteilnehmer gleich in zweifacher Hinsicht beitragen (Hollmann et al. 2010): Zum einen verhindert es ungewollte Wiederholungsqualifizierungen. Kompetenzen, die beispielsweise in einer Ausbildung erworben wurden, brauchen dann nicht mehr in einer nachgelagerten Qualifizie- rung wie etwa einem Studium oder einer Weiterbildung neu aufgebaut zu werden. Zum anderen eröffnet ein durchlässiges Bildungssystem den Durchstieg durch die unterschiedlichen Stufen des Systems. Insbesondere für die Hochschulen verbreitert sich die Basis, aus der potenzielle Studierende rekrutiert werden können. Angesichts der demografischen Entwicklung könnte dieser Effekt auch zur Nachwuchssicherung der Hochschulen beitragen.

Die Verknüpfung von Ausbildungsabschluss und Hochschulzugangsberechtigung hat trotz eines langen Vorlaufs bisher noch keine quantitative Bedeutung erlangt. Der Zugang von beruflich Qualifizierten ohne Hochschulzugangsberechtigung zu Hochschulen wird bisher nur in geringem Umfang von nicht mehr als $1 \%$ der $\mathrm{Zu}$ gangsberechtigten genutzt (BMBF 2007), obwohl bereits vor mehr als einem Jahrzehnt die Kultusministerkonferenz eine Vereinbarung über den Erwerb der Fachhochschulreife in beruflichen Bildungsgängen getroffen hatte, wonach berufliche Bildungsgänge in Abhängigkeit von den jeweiligen Bildungszielen und -inhalten sowie ihrer Dauer zu einer umfassenden Studierfähigkeit führen können. Demgegenüber ist das Potenzial aber beträchtlich, denn im Jahr 2008 gab es rund 240.000 Ausbildungsanfänger im dualen System, die über einen Realschul- oder gleichwertigen Abschluss verfügten.

Schnittstellenprobleme gibt es auch beim Übergang von Bachelor- zu Masterabschlüssen. Dies hängt auch damit zusammen, dass im deutschen Hochschulsystem verschiedene Formen von Masterstudiengängen möglich sind, bei denen ein Masterstudiengang als grundständiges Studium im Grundsatz dadurch ausgeschlossen ist, dass man ohne einen zuvor erworbenen berufsqualifizierenden Hochschulabschluss zu einem Masterabschluss gelangen kann (KMK 2010). Mit der Einführung des Bachelor- und Mastersystems hat sich eine kontrovers geführte Debatte über die Bedeutung und Relation von akademischer und beruflicher Bildung ergeben. Ursächlich trägt zu dieser Diskussion bei, dass im Gegensatz zu den Diplom- und Magisterabschlüssen die Bachelorabschlüsse nach den Strukturvorgaben der Kultusministerkonferenz explizit ein eigenständiges berufsqualifizierendes Profil aufweisen (KMK 2010) und bereits ab 180 Credit Points (vgl. Fußnote 5) akkreditiert werden. Dies entspricht einer
Regelstudienzeit von nur noch sechs Semestern, sodass sie eine zu einer dualen Berufsausbildung vergleichbare Dauer aufweisen (Hollmann et al. 2010).

Eine Konkurrenzsituation zwischen beruflich Qualifizierten und Akademikern kann sich demnach ergeben, wenn Bachelorsystem und Berufsbildungssystem miteinander in einen unmittelbaren Wettbewerb treten. In diesem Fall konkurrieren Bachelorabsolventen um Stellen, die klassisch von Absolventen mit einer geregelten Aufstiegsfortbildung (Meister, Techniker, Betriebswirte, etc.) besetzt werden. Diese Stellenprofile erfordern unter anderem eine breite berufliche Handlungskompetenz, ein tiefes Spezial- und großes Erfahrungswissen. Umgekehrt wäre denkbar, dass Absolventen einer beruflichen Ausund Weiterbildung um Stellen konkurrieren, die klassisch von Diplomabsolventen (FH) besetzt wurden. Dieser Fall könnte vermehrt eintreten, wenn Bachelorabsolventen keine vergleichbaren Kompetenzen zu Diplomabsolventen (FH) aufweisen und Facharbeiter insbesondere mit Aufstiegsfortbildung verstärkt in diese Stellen „hineinwachsen".

Andererseits ist auch ein Komplementärmodell denkbar, wenn die Schnittmengen geringer sind und eine Hochschulausbildung und eine berufliche Aus- und Weiterbildung nach wie vor zu unterschiedlichen Kompetenzen führen, die überwiegend verschiedenartige Qualifikationsbedarfe in der Wirtschaft abdecken. Bei dem derzeitigen Verbreitungsgrad von Bachelorabschlüssen muss die Frage empirisch derzeit aber noch unbeantwortet bleiben, ob eher das Konkurrenz- oder das Komplementärmodell realistisch ist. Wahrscheinlich wird es beide Tendenzen geben. Erste Befunde (Werner et al. 2008) deuten darauf hin, dass Akademiker aufgrund ihrer tendenziell höheren Entlohnung bei sonst ähnlichen Tätigkeitsmerkmalen in anderen Laufbahnen und Aufgabenfeldern tätig sind als Beschäftigte mit einer Aufstiegsweiterbildung.

Nicht zuletzt wegen der anhaltend hohen Nachfrage nach beiden Qualifikationsgruppen am Arbeitsmarkt ist wahrscheinlich, dass beide Systeme gleichermaßen benötigt werden. Zudem wird es das Ziel von Unternehmen bleiben, Fachkräfte ohne Zeitverlust höher zu qualifizieren sowohl innerhalb als auch außerhalb des Berufsbildungssystems. Durch eine größere formale Durchlässigkeit können per 
saldo zusätzliche Qualifikationspotenziale in Deutschland erschlossen werden. Dies betrifft gleichermaßen die Durchlässigkeit zwischen der dualen Berufsausbildung und der tertiären Bildung, der beruflichen Ausbildung und der Weiterbildung sowie das Verhältnis zwischen einem ersten berufsqualifizierenden Bachelor-Abschluss und den weiterführenden Masterabschlüssen.

\section{Neue Kooperationsformen von Hochschulen und Unternehmen}

Ein wesentliches Ziel der Bologna-Reform war es, einen ersten berufsbefähigenden Abschluss schneller als in der früheren Regelstudienzeit erreichen zu können. Dieses Ziel wird - bei allen Akzeptanzschwierigkeiten im Einzelnen - grundsätzlich erreicht. Die jetzt noch einmal seitens des Bundesministeriums für Bildung und Forschung (BMBF) angestrebten ergänzenden qualitätssichernden Maßnahmen insbesondere zur Verbesserung der Lehre werden darüber hinaus auch die Erreichung eines weiteren Ziels der Reform unterstützen, nämlich die Verkürzung der durchschnittlichen Verweildauer im Studium. Zwar sind in manchen Fächern die Abbruchraten spürbar zurückgegangen, doch ist im Durchschnitt die Quote mit $21 \%$ nur leicht rückläufig (Heublein et al. 2010). Doch während die Abbrüche in den Bachelor-Studiengängen heute durchschnittlich nach 2,3 Fachsemestern erfolgen, ist dies in den herkömmlichen Studiengängen erst nach 7,3 Fachsemestern der Fall gewesen. Die Reversibilität einer einmal getroffenen Studienfachentscheidung hat durch die gestuften Abschlüsse deutlich zugenommen.

Aus der Ursachenforschung zu den Abbrecherraten ist bekannt, dass unmittelbar nach den Leistungsproblemen und noch deutlich vor den als unzureichend empfundenen Studienbedingungen auch Finanzierungsprobleme eine Rolle spielen. Dies verweist auf die Notwendigkeit, bei der gesamten Studienfinanzierung noch deutlich stärker als bisher bedarfsorientiert vorzugehen. Der jetzt vorliegende Gesetzentwurf für ein bundesweites Nationales Stipendienprogramm sieht vor, dass durch eine Matching-Finanzierung je zur Hälfte aus privaten und aus öffentlichen Mitteln ein monatliches Stipendium in Höhe von $300 €$ nach Begabung und einkommensabhängig durch die Hochschulen vergeben werden soll.

Wie eine qualitative Studie zu Kooperationsformen von Hochschulen und Unternehmen ergeben hat (Konegen-Grenier 2009), werden unternehmensseitig Stipendien meist im Rahmen von bereits bestehenden Kooperationen mit Hochschulen vergeben. Unternehmensvertreter wenden sich häufig direkt an die Lehrstühle mit der Bitte um Empfehlung geeigneter Absolventen und stellen dabei Stipendien in Aussicht. Dabei wird die spätere Mitarbeit im Unternehmen relativ verbindlich abgesprochen. In manchen Fällen wird statt eines Stipendiums ein Darlehen gewährt, das dann bei späterer Mitarbeit nicht zurückgezahlt werden muss, oder es wird umgekehrt ein Stipendium gewährt, das zurückgezahlt werden muss, wenn sich der Studierende für ein anderes Unternehmen entscheidet.

Diese Vergabemuster erlauben die auch hochschulpolitisch relevante Beobachtung, dass bei der Stipendienvergabe der Aspekt der Mitarbeiterrekrutierung oder der Mitarbeiterförderung an erster Stelle steht. Die Erwartung, Unternehmen sollten sich allein aus allgemein gesellschaftspolitischen Motiven engagieren, ist wenig realistisch und bildungsökonomisch fehlspezifiziert. Vielmehr ist das Gewinnen von Fachkräften ein Katalysator des Unternehmensengagements: Die Bereitschaft, sich in der Studienfinanzierung zu engagieren, wächst mit dem Problemdruck, der durch fehlende Fachkräfte entsteht. Gut funktionierende Kooperationen erhöhen die Wahrscheinlichkeit, dass Unternehmen Stipendien vergeben. Das finanzielle Engagement für Studierende vertieft die Bindung des Unternehmens an die jeweilige Hochschule beziehungsweise an den jeweiligen Fachbereich.

Der weitere Verlauf der Hochschulreformen wird daher auch davon abhängen, ob und wie weit sich Hochschulen für Handlungsanreize öffnen, sich elementar um die Interessen ihrer Studierenden zu kümmern. Nur so lassen sich die Bedingungen dafür schaffen, dass die Lehre und die Betreuungsqualität für die Studierenden gestärkt werden können. Im Bereich der Forschung ist das Streben nach Anerkennung und Reputation in der jeweiligen scientific community traditionell stark verankert. In der Lehre hingegen gibt es bisher faktisch weder Drittmittel noch Preisgelder für Exzellenz. Nach bisheriger Gesetzeslage werden mit den Studienbeiträgen weniger als $10 \%$ des Lehretats nachfrageorientiert bewegt. Hinzu kommen im begrenztem Umfang Budgetveränderungen durch eine leistungs- und belastungsbezogene Mittelzuweisung der Landesministerien. Würde es gelingen, einen größeren Anteil nicht nur des Forschungs-, sondern auch des Lehretats im Wettbewerb zu vergeben, so erhielte auch die Entwicklung interner Steuerungsprozesse in den Hochschulen einen zusätzlichen Schub.

Die Studienreform hat aber auch noch eine bedeutsame weitere Implikation für die Kooperation zwischen Universitäten und Unternehmen. An den Hochschulen gewinnen neben den traditionellen Angeboten in Form von Seminaren und Kursen weiterbildende Studiengänge an Bedeutung, die auf einem ersten Hochschulabschluss aufbauen oder auf der Grundlage von beruflicher Ausbildung und Erfahrung zu einem ersten Hochschulgrad führen. Hier stellt sich die Aufgabe, den Zugang zu erleichtern und die Anrechnung bereits erworbener Kenntnisse und Kompetenzen $\mathrm{zu}$ verbessern. Internationale Vergleichsstudien zeigen, dass die wissenschaftliche Weiterbildung in Deutschland noch nicht den Stand anderer Industrienationen erreicht hat. Dies gilt insbesondere für die Position der Hochschulen auf dem Weiterbildungsmarkt (Schaeper et al. 2006).

Mit der Ermöglichung eines weiterbildenden Masterabschlusses eröffnet sich daher ein weites zusätzliches Feld universitärer Ausbildung in Form einer neuen dritten akademischen Abschlussform. Nach den ländergemeinsamen Strukturvorgaben für die Akkreditierung von Bachelor- und Masterstudiengängen sollen weiterbildende Masterstudiengänge an die berufliche Erfahrung anknüpfen und diese berücksichtigen. Neben einem qualifizierten Hochschulabschluss ist die Voraussetzung für den Zugang in der Regel eine mindestens einjährige qualifizierte Berufserfahrung. Die weiterbildenden Masterstudiengänge müssen daher den konsekutiven Masterstudiengängen ebenfalls in Bezug auf die Anforderungen gleichwertig sein.

Damit erhält auch das Thema Durchlässigkeit eine neue Qualität. Die viel zitierte Gleichwertigkeit, aber nicht Gleichartigkeit verschiedener Abschlüsse käme damit einen entscheidenden Schritt voran. Mit einem workload von 300 ECTS-Leis- 
tungspunkten ${ }^{5}$ sind die gleichen Anforderungen an den Weiterbildungs-Master wie bei den konsekutiven Studiengängen zu erfüllen. Insoweit erhält die Weiterbildung ein deutliches upgrading, ein berufsbegleitendes Studieren - auch und gerade im Fernstudium - wird deutlich attraktiver. Hochschulseitig kann die Modularisierung der Lehrinhalte damit einen zusätzlichen Schub erhalten. Auch die Verzahnung der grundständigen Hochschulausbildung mit der wissenschaftlichen Weiterbildung verlangt ein Mehr an Planungsaufwand, das aber durch zusätzliche Einnahmen gedeckt werden und überdies auch zu einem Profilierungsmerkmal für eine autonome Hochschule werden kann.

\section{6 \\ Fazit}

Alles in allem eröffnen die neuen gestuften Abschlüsse zusätzliche Realoptionen für die Studierenden, bieten eine Chance zur Verbesserung der Durchlässigkeit zwischen beruflicher und akademischer Qualifizierung, erhöhen die Bereitschaft von Unternehmen zur Ko-Finanzierung von Hochschulaktivitäten im Allgemeinen und zu Stipendien im Besonderen. Sie bieten zusätzliche Perspektiven für eine Universitätsstruktur im Wandel, die bei der Gestaltung ihrer Studienangebote die arbeitsweltgetriebene stärkere Verflech- tung von Bildungs- und Erwerbsphasen aufnimmt, flexible und zunehmend auch berufsbegleitende Studienangebote schafft und mit ihren Studienangeboten sowohl die Ziele der Persönlichkeitsentwicklung als auch der Beschäftigungsfähigkeit verfolgt. Insofern sind die gestuften Abschlüsse weit mehr als eine neue Studienorganisation, sondern sie sind Anstoß zu einem fundamentalen Wandel im Selbstverständnis von Hochschulen.

5 Das European Credit Transfer and Accumulation System (ECTS) soll sicherstellen, dass die Leistungen von Studenten an Hochschulen des europäischen Hochschulraums vergleichbar und bei einem Wechsel anrechenbar sind. Dies geschieht auf der Grundlage von Leistungspunkten (credit points).

\section{LITERATUR}

Anger, C./Plünnecke, A. (2009): Signalisiert die Akademikerlücke eine Lücke bei den Hochqualifizierten? - Deutschland und die USA im Vergleich, in: IW-Trends 3, S. 19-31

Bargel, T./Ramm, M./Multrus, F. (2010): Studiensituation und studentische Orientierungen, 10. Studierendensurvey an Universitäten und

Fachhochschulen, Berlin

Bundesministerium für Bildung und Forschung (BMBF) (2007):

Berufsbildungsbericht 2007, Bonn

Deutscher Industrie und Handelskammertag (DIHK) (2008): Die Studienreform zum Erfolg machen. Erwartungen der Wirtschaft an Hochschulabsolventen, Berlin

Eidgenössisches Staatssekretariat für Bildung und Forschung (2009): Internationales Ranking der Hochschulen, URL: http://www.sbf.admin. ch/htm/dokumentation/publikationen/grundlagen/factsheets/FS08_ ranking_d_nov_2009.pdf [Stand: 2010-03-17]

Franck, E./Opitz, C. (1999): Hochschulen als "Sortiereinrichtungen" in Humankapitalmärkten, in: Zeitschrift für Betriebswirtschaft 11, S. 1313-1330

Franck, E. /Opitz, C. (2004): Zur Filterleistung von Hochschulsystemen - Bildungswege von Topmanagern in den USA, Frankreich und Deutschland, in: Zeitschrift für betriebswirtschaftliche Forschung, Februar, S. 72-85

Heublein, U./Hutzsch, C./Schreiber, J./ Sommer, D./ Besuch, G. (2010): Ursachen des Studienabbruchs in Bachelor- und in herkömmlichen Studiengängen. Ergebnisse einer bundesweiten Befragung von Exmatrikulierten des Studienjahres 2007/08, HIS:Forum Hochschule 2, Hannover
Hochschulrektorenkonferenz (HRK) (2010): HRK-Präsidentin appelliert an Politik: Den vielen guten Worten nun endlich Taten folgen lassen, Pressemitteilung, 28.01.2010, Bonn

Hollmann, C./Neumann M./Schmidt, J./Werner, D. (2010): Perspektiven der dualen Berufsausbildung. Höhere Qualität und Effizienz durch stärkere Flexibilisierung und Durchlässigkeit, IW-Positionen 43, Köln Konegen-Grenier, C. (2009): Hochschulen und Wirtschaft. Formen der Kooperation und der Finanzierung, IW-Analysen 48, Köln

Konegen-Grenier, C./Koppel, O. (2009): Akzeptanz und Karrierechancen von Ingenieuren mit Bachelor- oder Masterabschluss, in: IW-Trends 4, S. $65-78$

Kultusministerkonferenz (KMK) (2009): Studium über berufliche Bildung. Wege und Berechtigungen, URL: http://www.kmk.org/ fileadmin/pdf/PresseUndAktuelles/2009/09-09_Hochschulzugang_ Berufliche_Bildung.pdf (Stand: 2010-01-28)

Kultusministerkonferenz (KMK) (2010): Ländergemeinsame Strukturvorgaben gemäß § 9 Abs. 2 HRG für die Akkreditierung von Bachelorund Masterstudiengängen, Beschluss der KMK vom 10.10.2003 i.d.F. vom 4.2.2010, Bonn

Meyer, D. (1990): Asymmetrische Information, Institutional Choice und die Funktion von Wertorientierungen, in: Jahrbuch für Sozialwissenschaft 1, Bd. 4, S. 104-121

Schaeper, H./Schramm, M./Weiland, M./Kraft, S./Wolter, A. (2006): International vergleichende Studie zur Teilnahme an Hochschulweiterbildung, in: HIS - Hochschul-Informations-System, Projektbericht, Oktober 2006, Hannover 
Schomburg, H./Teichler, U. (2009): Bachelor - Geht doch, in: Freie Universität Berlin, Beitrag zur Tagung "Studienbedingungen, Kompetenzerwerb und Berufserfolg ", 8.-9. Oktober, Berlin

Shapiro, C. (1983): Optimal pricing of experience goods, in: Bell Journal of Economics 2, S. 497-507

Stettes, O. (2006): Bildungsökonomische Grundlagen: Investitionen in Humankapital, in: Institut der deutschen Wirtschaft Köln (Hrsg.): Bildungsfinanzierung und Bildungsregulierung in Deutschland, IW-Studien, Köln, S. 31-59

St. Aubyn, M./Pina, A./Garcia, F./Pais. J. (2009): Study on the efficiency and effectiveness of public spending on tertiary education, in: Economic Papers 390, November, Brussels
Stiglitz, J. (1975): The Theory of "Screening ", Education and the Distribution of Income, in: American Economic Review 3, S. 283-300 Werner, D./Hollmann, C./Schmidt, J. (2008): Wie entwickeln sich angesichts des Strukturwandels zur Wissensgesellschaft und der Einführung der Bachelorstudiengänge die Chancen für duale Ausbildungsberufe und das duale System?, Abschlussbericht, URL: http:// www.iwkoeln.de/Portals/0/pdf/dokumente_andere/2009/dokumente_ wissensgesellschaft_studie.pdf [Stand: 2009-12-11] 